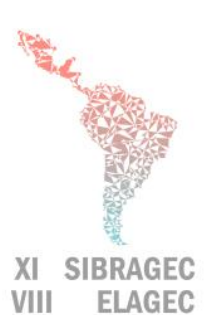

VIII ELAGEC
XI SIMPÓSIO BRASILEIRO DE GESTÃO E ECONOMIA DA CONSTRUÇÃO

VIII ENCUENTRO LATINOAMERICANO DE GESTIÓN

Y ECONOMÍA DE LA CONSTRUCCIÓN

Do conhecimento à ação: práticas avançadas de gestão da produção

Londrina, Paraná, Brasil. 23 a 25 de Outubro de 2019

\title{
A CONTRIBUIÇÃO DO BIM PARA ATENDIMENTO À NORMA DE DESEMPENHO - NBR 15575
}

\author{
RUBIM, Diana Fiori; CARVALHO, Aldo Ribeiro de (2); NUNES, Vitor Dias \\ Lopes (3); HIPPERT, Maria Aparecida Steinherz (4)
}

(1) Universidade Federal de Juiz de Fora - UFJF, (32) 98489-5787, e-mail:

diana.rubim@engenharia.ufjf.br (2) UFJF, e-mail: aldo.carvalho@engenharia.ufjf.br, (3) UFJF, e-mail: dias.vitor36@gmail.com, (4) UFJF, e-mail: aparecida.hippert@ufjf.edu.br

\begin{abstract}
The Performance Standard - NBR 15575, as well as ways to meet its requirements has been studied since its publication in 2013. Although many companies still found difficulties to verify compliance with these requirements, some studies aim to evaluate BIM's contribution to this task. This paper aims to verify among the studies already disclosed those dealing with the use of BIM. The search started by a Systematic Review of Literature (RSL), based on definitions of databases, search terms, deletion criteria and content analysis, returned a set of 9 papers on the topic. The results allow us to verify efforts to apply BIM for performance checking through the use of software. The point most highlighted by the researchers is the need for an automated process for checking the requirements of the Brazilian standard, as well as for creating a data library, increasing speed, and promoting the applicability of the same standard. However, the implementation of this routine still depends on companies making the transition to BIM as well as companies' greater awareness of meeting the performance standard.
\end{abstract}

Keywords: BIM, Performance, NBR 15575, Systematic literature review

\section{INTRODUÇÃO}

A norma de desempenho (ND), NBR 15575/2013, reflete as exigências dos usuários através de critérios e requisitos que objetivam garantir o desempenho das edificações habitacionais. Estes requisitos são capazes de promover a sustentabilidade, melhor custo benefício, aumento da vida útil, além de garantir um ambiente construído de qualidade e segurança para o usuário. Porém, muitas empresas ainda encontram dificuldades para verificação do atendimento a estes requisitos (Silva et al, 2014).

Por outro lado, surgem os recursos de Tecnologia da Informação que permitem auxiliar as empresas nesta tarefa, como o Building Information Modeling (BIM). Segundo Rodrigues (2015) o mesmo apresenta eficiência para verificação e analise de parâmetros normativos dentro de empreendimento, podendo potencializar os resultados do projeto.

O BIM, segundo Penttilã (2006), é uma metodologia que permite gerenciar a essência do projeto e dados do empreendimento, em formato digital, durante todo o ciclo de vida da construção. 


\section{SIBRAGEC - ELAGEC 2019 - de 23 a 25 de Outubro - LONDRINA - PR}

Neste sentido, o objetivo deste trabalho é verificar, dentre os estudos nacionais realizados sobre a ND, aqueles que abordam a contribuição do BIM para atendimento aos seus requisitos através de uma Revisão Sistemática da Literatura (RSL).

\subsection{Fundamentação Teórica}

A NBR 15575/2013 estabelece o conceito de desempenho por meio da definição de requisitos (qualitativos), critérios (quantitativos ou premissas) e métodos de avaliação, considerando a possibilidade de mensuração do seu cumprimento (ABNT, 2013). A norma define os critérios mínimos de desempenho para cada sistema da edificação e estabelece uma série de atribuições para todos os agentes da construção civil.

A NBR 15575 teve sua primeira versão publicada em 2008, passou por um processo de revisão pelas partes envolvidas, passando a vigorar em 2013. Silva et al (2014) apontaram que a implantação de forma integral da NBR 15575/2013 afetava projetistas, construtoras e fornecedores, que alegaram não ter tido tempo hábil para se adequar a normativa.

Por outro lado, dentre as tecnologias e métodos que podem facilitar a implantação da norma, nota-se, a contribuição da tecnologia BIM, conforme conclui Silva e Arantes (2017). O BIM é "uma tecnologia de modelagem e um grupo associado de processos para produção, comunicação e análise do modelo de construção" (EASTMAN et al., 2011). Para Freire, Marta e Sotelino (2015), a tecnologia BIM promove uma análise de informações de forma que quando ocorre alguma mudança em um dos componentes do projeto, a alteração é observada em todas as vistas do modelo, assim informando dados consistentes, não-redundantes e coordenados do empreendimento, e através desses conhecimentos é possível reduzir a ocorrência de erros e retrabalhos durante a execução do projeto.

\section{METODOLOGIA}

Para Randolph (2009) a RSL engloba a identificação da necessidade da revisão, definição da questão motivadora da pesquisa, além da elaboração do protocolo de busca, esta etapa trata do planejamento, a seguinte é a realização, que ocorre a aplicação dos critérios estabelecidos na primeira fase, a síntese e análise dos resultados. A terceira e última trata-se da divulgação dos resultados.

A RSL foi proposta com o objetivo de identificar, classificar e analisar os trabalhos referentes à ND de maneira a responder à seguinte questão motivadora: qual o panorama da produção científica referente à norma NBR 15575 desde o seu lançamento?

A RSL abrangeu as bases de dados: Periódicos da Coordenação de Aperfeiçoamento de Pessoal de Nível Superior (CAPES), Centro de Referência e Informação em Habitação (InfoHab), Catálogo de Teses e Dissertações da CAPES, Biblioteca Digital Brasileira de Teses e Dissertações (BDTD) e o Google Acadêmico, buscando conhecer, analisar e classificar os trabalhos relacionados à ND.

Inicialmente, definiu-se as palavras chaves da busca, utilizando o termo: "NBR 15575" ou "NBR 15.575". Utilizou-se o recorte temporal de 2008 a 2018, abrangendo desde a primeira versão da norma a data da pesquisa. Os arquivos deveriam ter acesso integral ao conteúdo, sendo válidos: artigos de anais de eventos, de periódicos, dissertações e teses. 
Em posse dos arquivos, observou-se nos títulos a presença do termo de pesquisa ou relação com as exigências dos usuários da ND (Segurança Estrutural; Segurança contra o fogo; Segurança no uso e na operação; Estanqueidade; Desempenho Térmico; Desempenho Acústico; Desempenho Lumínico; Saúde, Higiene e Qualidade do Ar; Funcionalidade e Acessibilidade; Conforto Tátil e Antropodinâmico; Durabilidade; Manutenibilidade; Impacto Ambiental; e Requisitos Gerais), lia-se, então, o resumo, em busca de relação direta com a NBR 15575. Após a aplicação dos filtros, dos 1342 resultados nas bases de dados restaram 329 trabalhos que discutem a ND.

$\mathrm{Na}$ segunda etapa, foi feita novamente a leitura dos títulos dos trabalhos da amostra final bem como das palavras chaves que deveriam conter o termo BIM ou Building Information Modeling sendo identificadas 9 pesquisas, as quais são analisadas e discutidas a seguir.

\section{RESULTADOS E DISCUSSÃO}

O BIM aplicado ao projeto pode ser visto em Coelho (2013), que explora o potencial da tecnologia, em etapas iniciais de projeto, para verificação de desempenho do projeto a partir de critérios da NBR 15575. Segundo Coelho (2013) a lógica BIM, aplicada pelo software Revit, possibilita a realização de algumas verificações de desempenho previstas na norma em uma interface orientada para arquitetos fornecida pelo software, o que contribui para a incorporação do conceito de desempenho, podendo ainda ampliar a avaliação a partir da interoperabilidade com outras ferramentas de simulação.

Silva (2017) e Silva e Arantes (2017) analisam a viabilidade e obstáculos da automatização para verificação dos requisitos da ND, utilizando o software Solibri Model Checker para modelos em BIM. Para tal, eles afirmam ser necessário cumprir algumas etapas. Primeiro na padronização das diretrizes de modelagem e adoção dos sistemas de classificação da informação da construção, as quais precisam atender os requisitos da norma, e por fim, pela construção da parametrização das regras preliminares e regras principais. Recomenda-se a realização de múltiplos testes em modelos para o aperfeiçoamento da proposta de parametrização das regras. O estudo mostrou uma redução de tempo de conferência de projeto em $60 \%$, e apontou $22 \%$ de não conformidades a mais do que as encontradas manualmente, fato que comprova a aplicabilidade da verificação computacional. Ademais, um terço dos requisitos da norma são passíveis de checagem automática. Como desafio encontrado está a subjetividade de $42 \%$ dos requisitos, situação que dificulta a criação de um sistema que dispense a interação humana.

Ainda relacionando desempenho e projeto, Silva Junior (2016) estudou métodos de projeto de arquitetura e suas fases, e encontrou no BIM uma evolução em relação ao processo tradicional. O estudo mostrou que o uso de parâmetros de desempenho ao modelo gera resultados positivos, sendo alguns desses a identificação de requisitos e recursos correlatos existentes no software, gestão e organização de informações e a possibilidade de informar e identificar um terço dos requisitos impostos pela ND. Também observou que a criação de uma biblioteca de componentes com informações padronizadas otimiza o trabalho do projetista.

A questão da biblioteca de componentes é tratada por Nardelli e Oliveira (2013), que discutem a problemática da adaptação e ampliação da biblioteca fornecida pelo Ministério do Desenvolvimento, Indústria e Comércio Exterior, a partir da vigência da NBR 15575/2013, considerando uma edificação do Programa Minha Casa Minha Vida 


\section{SIBRAGEC - ELAGEC 2019 - de 23 a 25 de Outubro - LONDRINA - PR}

(PMCMV). Identificou-se a necessidade dos parâmetros da norma serem verificados ainda na fase de projeto, e para tal, um facilitador seria a criação de uma biblioteca de componentes BIM, com os atributos necessários para realização de simulações. $\mathrm{O}$ primeiro problema identificado é que os elementos que constituem as famílias fornecidas para o programa Revit não possuem parâmetros bem definidos e completos, pois faltam informações sobre os componentes e materiais dos elementos presentes nas famílias. Os autores declaram que somente com dados sólidos será possível fazer uma análise da ND e estimar o custo total por unidade, sendo esse um critério decisivo na construção de Habitações de Interesse Social (HIS).

Por meio do estudo das construções do PMCMV, Tonso e Nardelli (2015) analisam a aplicação do BIM para verificação de desempenho térmico com base na ND. A análise térmica realizada baseou-se nas propriedades dos materiais em um modelo no Revit. Os autores retratam a dificuldade de inserção de tantos parâmetros manualmente e completam dizendo que o uso do BIM deveria entrar nesse contexto, visando a criação de bancos de dados e informações, os quais gerariam agilidade ao processo de análise e ofereceriam projetos com mais informações, consistência e menos erros. Otrabalho mostra, também, a evolução da tecnologia em relação a Pires et al. (2012), o qual possui mesmos objetivos, complementado pela ferramenta Energy Plus; os autores criticaram a grande complexidade dos modelos e dificuldade de trabalhar com as famílias e associar com simulações.

Silva et al. (2017) propõem uma rotina de trabalho no Revit utilizando o plug-in Dynamo, visando avaliar o desempenho acústico de paredes de divisória, baseando-se na NBR 15575. Os esforços estão sendo feitos para que ocorra uma verificação automática dos critérios, bem como em Silva (2017). Os autores desejam a aplicação do mesmo em etapas iniciais do projeto, direcionada para a escolha de esquadrias e elementos de vedação, otimizando a performance acústica dos projetos.

Para mensurar os custos de uma edificação térrea de HIS e seu aumento no valor da construção quando regida pelos critérios da ND, Dantas Filho et al (2014) fizeram uma análise através de um modelo virtual em BIM. Verificou-seque o custo geral é $12 \%$ mais alto que os de projetos tradicionais, evidenciando que o maior aumento foi no sistema de cobertura, em uma porcentagem aproximada de $60 \%$, constataram, também, que estes aumentos são considerados altos e podem inviabilizar economicamente os investimentos em HIS apresentados pelo governo brasileiro.

\section{CONSIDERAÇÕES FINAIS}

A partir da análise dos trabalhos selecionados da RSL, é possível verificar esforços para a aplicação do BIM para checagem de desempenho por meio de verificações em interfaces fornecidas por softwares, como o Revit ou outros programas. Os autores sugerem a criação de banco de dados padronizados de maneira a promover agilidade para o processo.

A problemática que envolve a efetivação dessa rotina é que além das empresas não terem realizado a transição para metodologia BIM, muitas não realizam de fato tais verificações. Um agravante destacado é a subjetividade de alguns requisitos da norma, fazendo necessária ainda a interação humana.

Com a união de esforços da academia, para formação de profissionais com conhecimento das ferramentas BIM, somada à uma maior conscientização das empresas sobre a necessidade de atendimento da ND, será possível melhorar o desempenho das 
SIBRAGEC - ELAGEC 2019 - de 23 a 25 de Outubro - LONDRINA - PR

edificações brasileiras e promover produtos de maior qualidade para o setor da construção civil.

\section{REFERÊNCIAS}

ASSOCIAÇÃO BRASILEIRA DE NORMAS TÉCNICAS (ABNT). NBR 15575: Edificações habitacionais - Desempenho. Rio de Janeiro, 2013.

COELHO, F. F. de M. O potencial e limitações do BIM - Building Information Modeling para o atendimento da ABNT NBR 15575. 2013. Dissertação (Mestrado em Concentração: Projeto, Produção e Gestão do edifício) - Programa de Pós-Graduação em Arquitetura e Urbanismo, Universidade Federal Fluminense, Niterói, 2013.

DANTAS FILHO, J. B. P.; GUEDES, J. P.; CÂNDIDO, L. F.; BERTINI, A. A.; NETO, J. L. T. The costs of the performance standards in a social housing using the BIM platform. In: IAHS WORLD CONGRESS ON HOUSING, 40., Funchal, 2014. Anais [...]. Funchal, 2014.

EASTMAN, C.; TEICHOLZ, P.; SACKS, R.; LISTON, K. BIM Handbook: a guide to Building Information Modeling for owners, managers, designers, engineers, and contractors. Hoboken, Nova Jérsei, EUA: John Wiley \&Amp; Sons, 2. ed., 2011.

NARDELLI, E. S.; OLIVEIRA, J. T. BIM e Desempenho no Programa Minha Casa Minha Vida - PMCMV. In: CONGRESO DE LA SOCIEDAD IBEROAMERICANA DE GRÁFICA DIGITAL EN VALPARAÍSO, 17., Santa Maria, 2013. Anais [...]. Sigrad: Santa Maria, 2013.

PENTTILÄ, H. Describing the changes in architectural Information Technology to understand design complexity and free-form architectural expression. Journal of Information Technology in Construction, v. 11, Special Issue, p. 395-408, 2006.

RANDOLPH, J. J. A guide to writing the dissertation literature review. Practical Assessment, Research \& Evaluation, v. 14, n. 11, p. 1-13, jun. 2009.

SILVA, A. T.; KERN, A. P.; PICCOLI, R.; GONZÁLEZ, M. A. S. Novas exigências decorrentes de programas de certificação ambiental de prédios e de normas de desempenho na construção. Arquitetura Revista, São Leopoldo, v.10, n. 2, p. 105-114, jul./dez. 2014.

SILVA, F. A.; ARANTES, E. Verificação automática de requisitos de projetos da Norma de Desempenho NBR 15575 a partir da adequação de regras da plataforma BIM Solibri Model Checker. In: SIMPÓSIO BRASILEIRO DE TECNOLOGIA DA INFORMAÇÃO E COMUNICAÇÃO NA CONSTRUÇÃO 1.; SIMPÓSIO BRASILEIRO DE GESTÃO E ECONOMIA DA CONSTRUÇÃO 10., Fortaleza, 2017. Anais [...]. Porto Alegre: Antac, 2017.

SILVA, F. P. de A. Verificação automática dos requisitos de projetos da Norma De Desempenho pela plataforma BIM Solibri Model Checker. 2017. Dissertação (Mestrado em Construção Civil). Escola de Engenharia, Universidade Federal de Minas Gerais, Belo Horizonte, 2017.

SILVA JÚNIOR, M. A. Parâmetros de desempenho incorporados em projetos de arquitetura com o uso de aplicativo de modelagem BIM. 2016. Dissertação (Mestrado em Habitação: Planejamento e Tecnologia) - Instituto de Pesquisas Tecnológicas do Estado de São Paulo, São Paulo, 2016.

SILVA, J. L.; MUSSI, A. Q.; RIBEIRO, L. A.; SILVA, T. L. Programação em plataforma BIM e a Norma de Desempenho Brasileira: Desenvolvimento de uma aplicação para estimativa de performance acústica em projetos arquitetônicos. Congreso de la sociedad ibero-americana de gráfica digital, 21., 2017, Concepción. Anais [...]. Concepcíon: Sigrad, 2017.

TONSO, L. G.; NARDELLI, E. S. BIM para a análise de desempenho térmico em edificações do Programa Minha Casa Minha Vida. In: CONGRESSO DA SOCIEDADE IBERO-

AMERICANA DE GRÁFICA DIGITAL, 19., Florianópolis, 2015. Anais [...]. Sigrad:

Florianópolis, 2015. 
SIBRAGEC - ELAGEC 2019 - de 23 a 25 de Outubro - LONDRINA - PR

AGRADECIMENTOS

À UFJF e à CAPES, pelo apoio recebido. 